\title{
Ecoulement engendré par la houle dans un milieu poreux
}

\author{
Brossard J ${ }^{(a)}$, Chagdali M. ${ }^{(b)}$, Rey V. ${ }^{(c)}$ \\ ${ }^{(a)} M C F, H D R, L M P G$, université du Havre, brossard@univ-lehavre.fr \\ ${ }^{(b)}$ Professeur, LCSM, université Hassan II, Csablanca, m_chagdali@hotmail.com

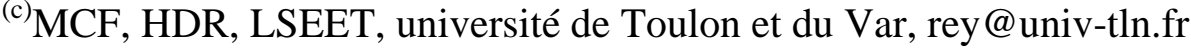

\section{Résumé}

Le modèle de Polubarinova-Kochina est utilisé pour décrire l'écoulement instationnaire engendré par la houle dans un milieu poreux. Ce modèle introduit des coefficients, notamment de masse ajoutée et de perméabilité. Les expériences réalisées en canal à houle avec une mesure très précise de la surface libre permettent de quantifier ces coefficients. Les champs de vitesse dans le milieu externe et dans le milieu poreux peuvent alors être déterminés. Ceux-ci montrent une discontinuité et un saut de phase pour la composante horizontale dus aux conditions aux limites imposées à l'interface poreux/milieu externe. Une amélioration dans la description nécessiterait une modélisation de type couche limite à l'interface.

\begin{abstract}
$\underline{\text { Abstract }}$
The Polubarinova-Kochina theoretical formulation is used to describe the non sationary flow induced by propagative wave into a porous medium. In this model, some parameters, as permeability or virtual mass coefficient, are used to model the resistance forces. Experiments carried out in a wave flume and an accurate measurement method allow to quantify these cefficients. The flow fields are obtained from these results. The vertical distribution of the horizontal component of the flow exhibits a discontinuity and a phase shift at the interface of the porous medium. An improvement should be to introduce a viscous model for the boundary conditions at this interface.
\end{abstract}

Mots clés:milieu poreux, houle, modèle de Polubarinova-Kochina, fonds sédimentaires

\section{Introduction}

Certains fonds marins peuvent être considérés comme des milieux poreux; ainsi les massifs coralliens, les fonds recouverts d'une flore abondante et dense, les couches sédimentaires constituées de grosses «particules» comme des galets ou des graviers. La perméabilité de ces milieux induit un écoulement interne et des flux non négligeables au travers l'interface milieu poreux-milieu externe lorsque se propage au dessus une houle.

Comme toute interface, la frontière entre le milieu externe et le milieu poreux est une zone extrêmement sensible à cause des gradients de différentes grandeurs physiques comme la vitesse, la pression, etc... Ces zones de gradient sont favorables au développement de la vie végétale et animale ; mais elles sont très sensibles à la pollution. Celle-ci peut être due à des contaminants dissous, à des particules solides comme des métaux lourds, à des grains sédimentaires enrobés de polluant comme du pétrole ou transportant d'infimes particules radioactives.

Afin de déterminer les caractéristiques hydrodynamiques du milieu poreux nous avons utilisé le modèle Polubarinova-Kochina adapté aux écoulements instationnaires. Ce modèle introduit des paramètres comme un coefficient de masse ajoutée et un coefficient de traînée turbulente qui sont très difficiles à déterminer par des mesures internes au milieu poreux. En 
utilisant la technique de mesure de surface libre par effet Doppler ${ }^{2}$, nous avons pu déterminer ces coefficients par les mesures de la longueur d'onde et du coefficient d'amortissement.

\section{$\underline{\text { 2.Modèle théorique d'écoulement instationnaire dans un milieu poreux }}$}

Nous supposons que dans la zone externe de hauteur $h_{e}$ l'écoulement est irrotationnel, d'où l'existence d'un potentiel des vitesses e. En homogénéisant le milieu poreux on peut introduire un potentiel de vitesse $\mathrm{p}$. Il reste à résoudre l'équation de Laplace en prenant en compte les conditions aux interfaces.

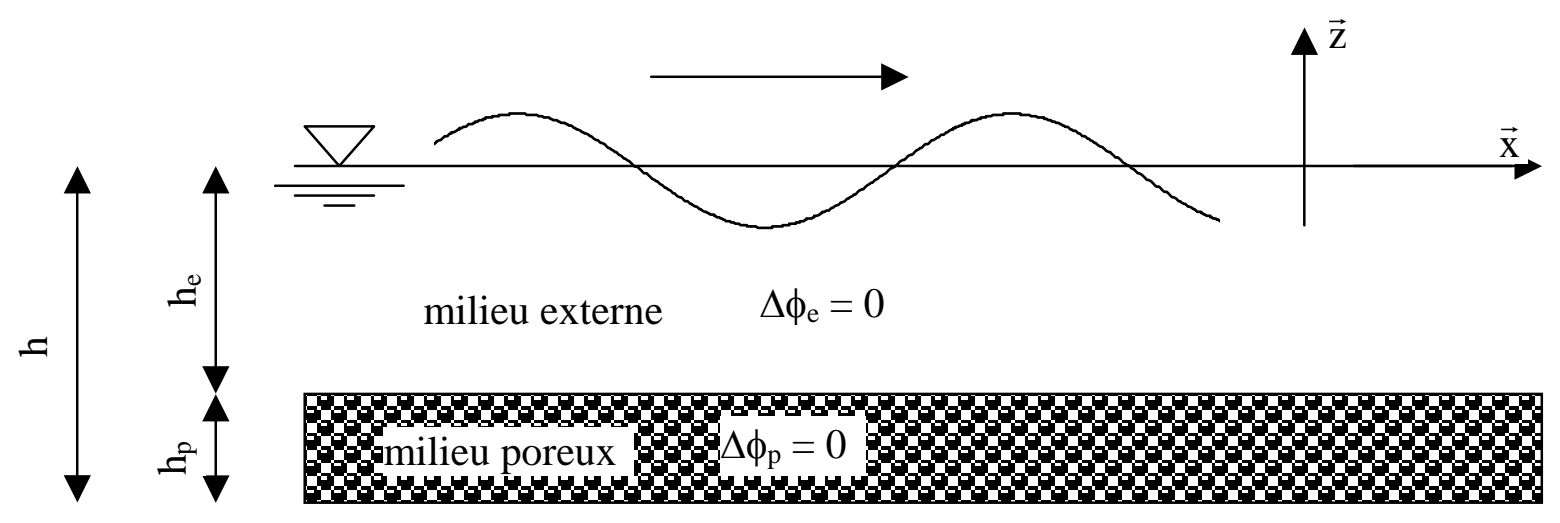

Figure 1 - Configuration théorique

La méthode utilisée est celle développée par Sollitt et Cross $^{3}$.

A l'échelle du pore, l'équation de Navier Stokes peut s'écrire :

$$
\frac{\partial \overrightarrow{\mathrm{q}}}{\partial \mathrm{t}}+\frac{1}{2} \overrightarrow{\operatorname{grad}} \mathrm{q}^{2}=-\frac{1}{\rho} \overrightarrow{\operatorname{grad}} \mathrm{p}_{\mathrm{p}}-\mathrm{g} \overrightarrow{\mathrm{z}}+\overrightarrow{\mathrm{Fr}}
$$

où $\vec{q}$ est une vitesse moyenne à l'échelle du pore et $\overrightarrow{F r}$ les forces résistantes à l'échelle du pore.

Le modèle théorique de Polubarinova-Kochina permet d'expliciter les forces résistantes à l'échelle macroscopique sous la forme :

$$
-\frac{v}{\mathrm{~K}} \overrightarrow{\mathrm{u}}-\frac{\mathrm{C}_{\mathrm{f}}}{\sqrt{\mathrm{K}}}|\overrightarrow{\mathrm{u}}| \overrightarrow{\mathrm{u}}-\frac{1-\mathrm{n}}{\mathrm{n}^{2}} \mathrm{C}_{\mathrm{m}} \frac{\partial \overrightarrow{\mathrm{u}}}{\partial \mathrm{t}}
$$

où $n$ est la porosité, $K$ la perméabilité intrinsèque, la viscosité cinématique et $\overrightarrow{\mathrm{u}}=\mathrm{n} \overrightarrow{\mathrm{q}}$.

La perméabilité intrinsèque peut être définie par l'expression :

$$
K=\frac{n^{2} d^{2}}{\alpha(1-n)^{3}}
$$

où $\mathrm{d}$ est le diamètre des particules et est un coefficient de tortuosité.

Le premier terme, linéaire, est le terme de Darcy. Le deuxième terme, non linéaire, est associé soit aux termes convectifs (à l'échelle du pore) soit à la turbulence; on note $\mathrm{C}_{\mathrm{f}}$ le coefficient de frottement. Le dernier terme est un terme d'inertie associé à l'instationnarité de l'écoulement, il fait intervenir un coefficient de masse ajoutée $C_{m}$.

L'équation (1) moyennée à l'échelle macroscopique devient :

$$
\mathrm{S} \frac{\partial \overrightarrow{\mathrm{u}}}{\partial \mathrm{t}}+\frac{1}{2} \frac{1}{\mathrm{n}^{2}} \overrightarrow{\operatorname{grad}} \mathrm{u}^{2}=-\frac{1}{\rho} \overrightarrow{\operatorname{grad}} \mathrm{p}_{\mathrm{p}}-\mathrm{g} \overrightarrow{\mathrm{z}}-\left(\frac{\mathrm{v}}{\mathrm{K}}+\frac{\mathrm{C}_{\mathrm{f}}}{\sqrt{\mathrm{K}}}|\overrightarrow{\mathrm{u}}|\right) \overrightarrow{\mathrm{u}}
$$


où $\mathrm{S}=\frac{1}{\mathrm{n}}+\frac{1-\mathrm{n}}{\mathrm{n}^{2}} \mathrm{C}_{\mathrm{m}}$ avec $\mathrm{C}_{\mathrm{m}}$ coefficient de masse ajoutée associé aux particules constituant le milieu poreux granulaire.

La linéarisation de l'équation (2) peut s'obtenir en négligeant le terme convectif (à l'échelle du milieu homogénéisé) et en utilisant la procédure de Lorentz qui consiste à remplacer le terme de dissipation par l'expression linéaire :

$$
\left(\frac{v}{K}+\frac{C_{f}}{\sqrt{K}}|\vec{u}|\right) \overrightarrow{\mathrm{u}}=\mathrm{f} \omega \overrightarrow{\mathrm{u}}
$$

où $f$ est un coefficient de dissipation.

$\mathrm{Gu}$ et $\mathrm{Wang}^{4}$, proposent une méthode d'itération s'appuyant sur l'intégration d'un flux d'énergie sur un contour, flux moyenné sur une période.

Dans notre cas nous supposerons que le caractère instationnaire de l'écoulement est prépondérant par rapport au caractère non linéaire ; d'où :

$$
\mathrm{f}=\frac{v}{\omega} \frac{1}{\mathrm{~K}} \text {. }
$$

L'équation (2) linéarisée devient, en remplaçant $\overrightarrow{\mathrm{u}}$ par $\overrightarrow{\operatorname{grad}} \phi_{\mathrm{p}}$ :

$$
\mathrm{S} \frac{\partial \phi_{\mathrm{p}}}{\partial \mathrm{t}}+\frac{\mathrm{p}_{\mathrm{p}}}{\rho}+\mathrm{gz}+\mathrm{f} \omega \phi_{\mathrm{p}}=0
$$

Pour le milieu fluide externe l'équation de Bernoulli linéarisée est :

$$
\frac{\partial \phi_{\mathrm{e}}}{\partial \mathrm{t}}+\frac{\mathrm{p}_{\mathrm{e}}}{\rho}+\mathrm{gz}=0
$$

$\mathrm{p}_{\mathrm{e}}$ et $\mathrm{p}_{\mathrm{p}}$ sont les pressions relatives régnant dans le milieu externe et dans le milieu poreux respectivement.

Le système d'équations à résoudre est :

$\mid \begin{aligned} & \Delta \phi_{\mathrm{e}}=0 \\ & \Delta \phi_{\mathrm{p}}=0\end{aligned} \quad$ avec les conditions aux limites linéarisées suivantes :

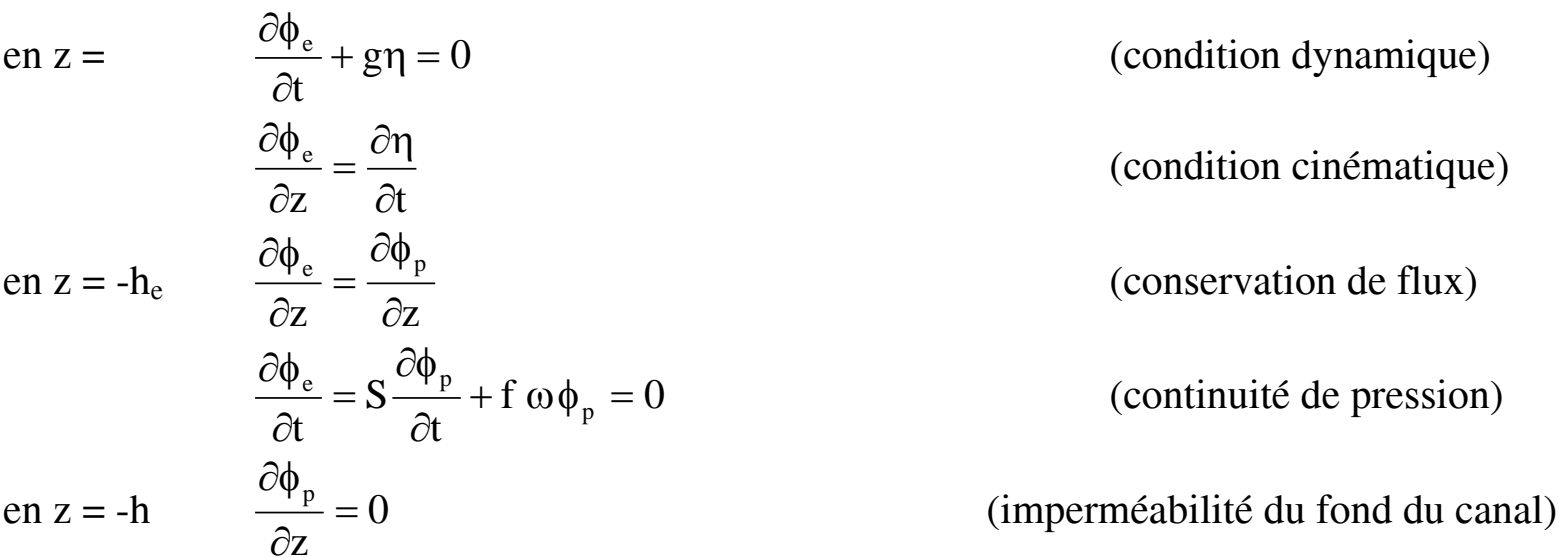

Ce système admet une solution d'onde propagative telle que :

$$
\begin{aligned}
& \phi_{\mathrm{e}}=\mathrm{i} \frac{\mathrm{a}}{\mathrm{k} \omega}\left[\omega^{2} \sinh (\mathrm{kz})+\mathrm{gk} \cosh (\mathrm{kz})\right] \mathrm{e}^{\mathrm{i}(\omega t-\mathrm{kx})} \\
& \phi_{\mathrm{p}}=\mathrm{i} \frac{\mathrm{a}}{\mathrm{k} \omega} \cosh [\mathrm{k}(\mathrm{z}+\mathrm{h})]\left[\frac{\omega^{2} \cosh \left(\mathrm{kh} \mathrm{e}_{\mathrm{e}}\right)-\mathrm{gk} \sinh \left(\mathrm{kh} \mathrm{e}_{\mathrm{e}}\right)}{\sinh \left(\mathrm{kh} \mathrm{p}_{\mathrm{p}}\right)}\right] \mathrm{e}^{\mathrm{i}(\omega \mathrm{t}-\mathrm{kx})}
\end{aligned}
$$


où $\mathrm{k}$ est le nombre d'onde complexe tel que $\mathrm{k}=\mathrm{k}_{\mathrm{p}}+\mathrm{i}_{\mathrm{a}}$ avec la partie réelle $\mathrm{k}_{\mathrm{p}}$ nombre d'onde et la partie imaginaire $\mathrm{k}_{\mathrm{a}}$ le coefficient d'amortissement ; la pulsation, a l'amplitude de l'onde de surface en $\mathrm{x}=0$.

Le nombre d'onde $\mathrm{k}$ vérifie l'équation de dispersion :

$$
\omega^{2}=\frac{(\mathrm{f}+\mathrm{iS}) g k \tanh \left(\mathrm{kh}_{\mathrm{e}}\right)+\operatorname{igk} \tanh \left(\mathrm{kh}_{\mathrm{p}}\right)}{(\mathrm{f}+\mathrm{iS})+\mathrm{i} \tanh \left(\mathrm{kh}_{\mathrm{e}}\right) \tanh \left(\mathrm{kh}_{\mathrm{p}}\right)}
$$

et la forme de la surface libre est :

$$
\eta(x, t)=a e^{k_{a} x} e^{i\left(\omega t-k_{p} x\right)}
$$

Les essais que nous réalisons en canal à houle avec un certain milieu poreux nous permettent de déterminer $\mathrm{k}_{\mathrm{p}}$ et $\mathrm{k}_{\mathrm{a}}$ pour différentes fréquences angulaires ; il reste ensuite à ajuster les coefficients $\mathrm{S}$ et $\mathrm{f}$ pour que les courbes d'évolution du coefficient d'amortissement en fonction de la fréquence déduit de l'équation de dispersion précédente correspondent au mieux avec les données expérimentales.

\section{Configuration expérimentale}

Les essais ont été réalisés dans un canal à houle régulière de $10 \mathrm{~m}$ de long et $0.3 \mathrm{~m}$ de large. Le système de mesure de surface libre permet de mesurer indépendamment les fréquences, les nombres d'onde et les coefficients d'amortissement des différents modes.

Le fond poreux est réalisé avec des billes de verre de diamètre $9 \mathrm{~mm}$. Nous avons testé deux épaisseurs de fond poreux. Dans le premier cas le fond poreux est constitué de 5 couches de billes avec une distance estimée entre le fond du canal et les centres des billes supérieures de $30 \mathrm{~mm}$ et dans le deuxième cas il est constitué de 7 couches soit une distance estimée entre le fond du canal et les centres des billes supérieures de $50 \mathrm{~mm}$.

Pour prendre en compte la dissipation induite par les « aspérités » de la couche supérieure nous avons réalisé des mesures de coefficient d'amortissement pour une seule couche de billes. Les valeurs obtenues ont été soustraites à celles obtenues pour les deux épaisseurs 30 et $50 \mathrm{~mm}$. Cette méthode permet aussi de prendre en compte les couches limites se développant sur les parois latérales.

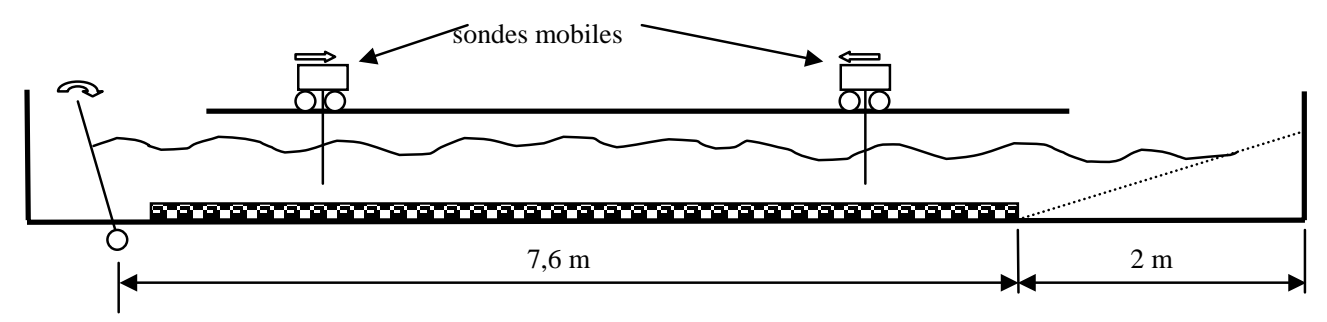

Figure 2 - Configuration expérimentale

Nous avons testé plusieurs valeurs de hauteur $h_{\mathrm{e}}$ pour le milieu fluide externe. De même nous avons testé plusieurs valeurs de la fréquence $\mathrm{F}$ de la houle comprises entre 0.8 et $1.5 \mathrm{~Hz}$. C'est sur ces évolutions du coefficient d'amortissement en fonction de la fréquence que nous déduisons les caractéristiques du milieu poreux. 


\section{$\underline{\text { 4.Résultats de mesure des longueurs d'onde }}$}

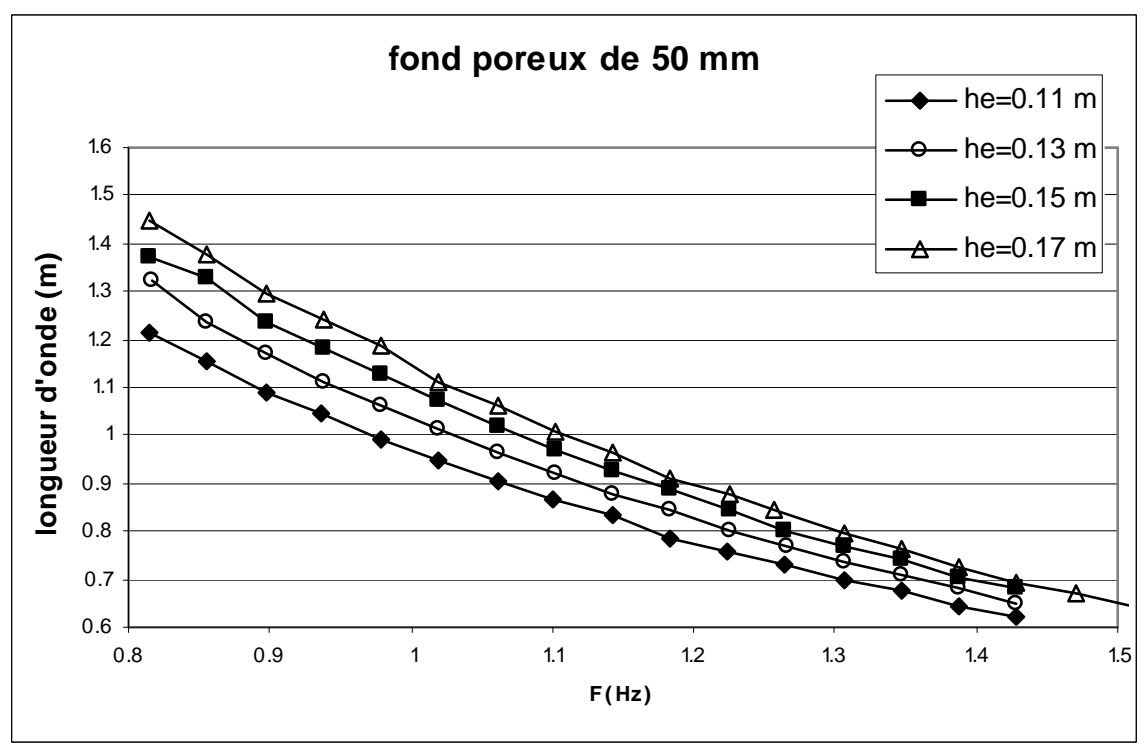

Figure 3 - Longueurs d'onde mesurées

Sur la figure 3 sont représentées (à titre d'exemple) les évolutions de la longueur d'onde mesurée pour une épaisseur de $50 \mathrm{~mm}$ en fonction de la fréquence de la houle. Les évolutions sont semblables dans tous les cas avec, classiquement, une décroissance en fonction de la hauteur d'eau au dessus du milieu poreux.

La longueur d'onde croît légèrement quand le milieu poreux devient plus épais.

\section{Coefficients d'amortissement}

Pour chaque essai l'amortissement est déterminé par la procédure de Hilbert appliqué au signal de surface libre enregistré par la sonde mobile. Cette méthode permet d'obtenir l'évolution de l'amplitude suivant $\mathrm{x}$ dont on déduit le coefficient d'amortissement.

La figure 4 présente les résultats expérimentaux obtenus.

Pour déterminer le coefficient d'inertie $\mathrm{S}$ et la perméabilité $\mathrm{K}$ du milieu nous résolvons l'équation de dispersion (5) en itérant sur les valeurs de $\mathrm{S}$ et $\mathrm{K}$ pour que les évolutions théoriques en fonction de la fréquence ajustent au mieux les évolutions expérimentales. Avec ces valeurs optimales des coefficients nous obtenons les évolutions théoriques présentées aussi sur la figure 4. Sur cette figure on observe que l'amortissement croît lorsque le rapport $\mathrm{h}_{\mathrm{p}} / \mathrm{h}_{\mathrm{e}}$ croît quelque soit la fréquence, ce qui était attendu intuitivement. Par contre on remarque que la variation de l'amortissement en fonction de la fréquence n'est pas monotone, ceci est prévu par la théorie mais est beaucoup moins intuitif que le phénomène précédent. 

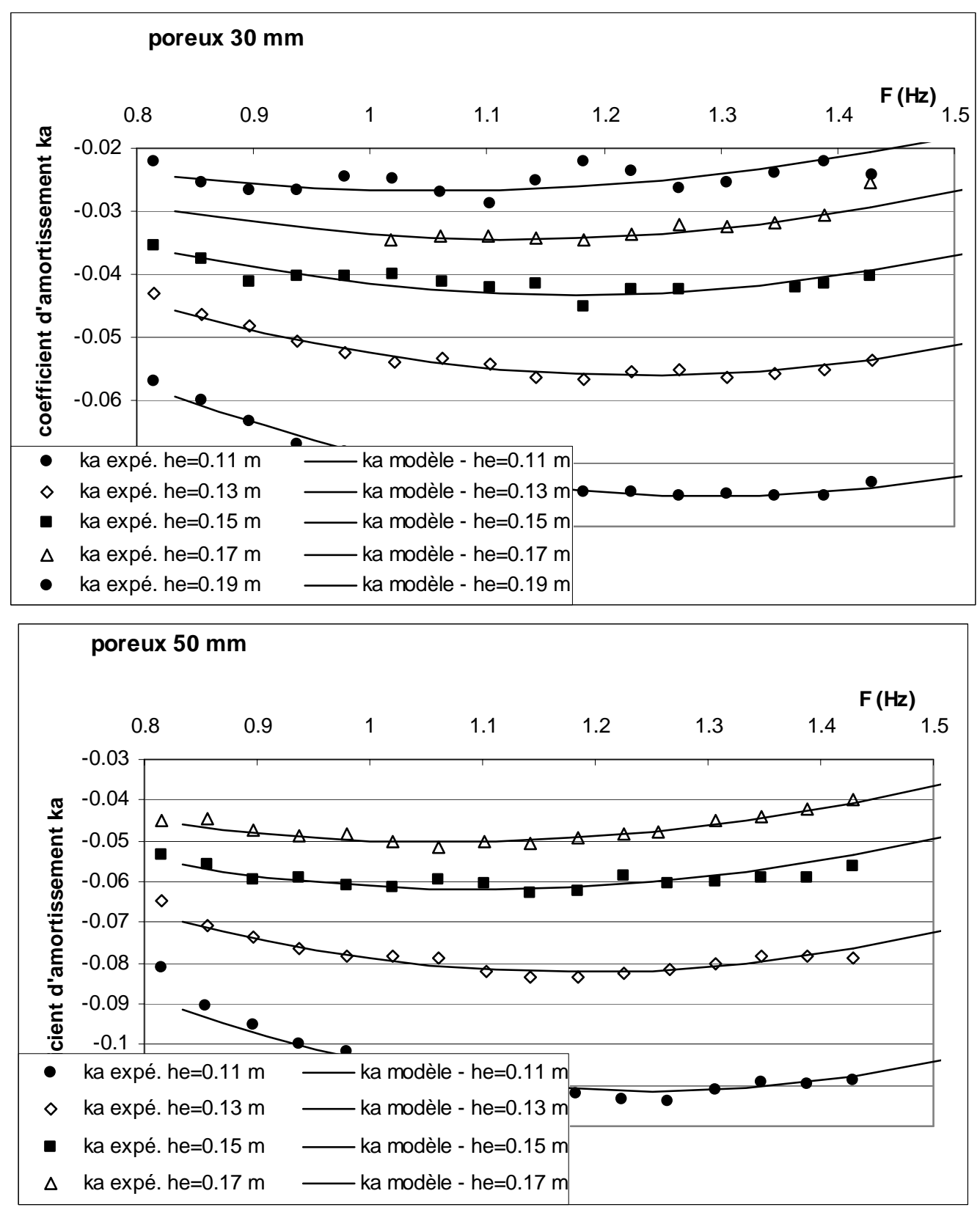

Figure 4-Coefficients d'amortissement expérimentaux et approximation théorique

\section{Caractéristiques du milieu poreux}

Le tableau 1 suivant donne les valeurs de la perméabilité $\mathrm{K}$ et du coefficient d'instationnarité $\mathrm{S}$ déduits de l'ajustement $\mathrm{du}$ modèle théorique avec les données expérimentales.

La porosité $n$ a été estimée par des expériences appropriées à 0,42. Des valeurs connues de $\mathrm{d}$, diamètre des billes et des valeurs estimées de $\mathrm{n}, \mathrm{K}$ et $\mathrm{S}$ nous pouvons déduire les valeurs du coefficient de masse ajoutée $\mathrm{Cm}$ et du coefficient de tortuosité . Pour ce dernier nous retrouvons les valeurs empiriques déterminées par Engelund ${ }^{5}$ et généralement admises. 
Tableau 1 - Caractéristiques du milieu poreux déduites des mesures de surface libre

\begin{tabular}{|c|c|c|c|c|c|}
\hline & & perméabilité & $\begin{array}{c}\text { coefficient } \\
\text { d'instationnarité }\end{array}$ & $\begin{array}{l}\text { coefficient de } \\
\text { masse ajoutée }\end{array}$ & $\begin{array}{l}\text { coefficient de } \\
\text { tortuosité }\end{array}$ \\
\hline & hauteur d'eau & $\mathbf{K}\left(\mathbf{m}^{2}\right)$ & $\mathbf{S}$ & $\mathrm{Cm}$ & a0 \\
\hline \multirow{5}{*}{$\begin{array}{l}\text { épaisseur de } \\
\text { poreux } 30 \mathrm{~mm}\end{array}$} & he $=0.110$ & $3.24 \mathrm{E}-08$ & 5.15 & 0.86 & 2306 \\
\hline & he $=0.130$ & $3.24 \mathrm{E}-08$ & 5.15 & 0.86 & 2306 \\
\hline & he $=0.150$ & $3.24 \mathrm{E}-08$ & 5.15 & 0.86 & 2306 \\
\hline & he $=0.170$ & $3.24 \mathrm{E}-08$ & 5.15 & 0.86 & 2306 \\
\hline & he $=0.190$ & $3.44 \mathrm{E}-08$ & 5.3 & 0.90 & 2170 \\
\hline \multirow{4}{*}{$\begin{array}{l}\text { épaisseur de } \\
\text { poreux } 50 \mathrm{~mm}\end{array}$} & $h e=0.110$ & $3.14 \mathrm{E}-08$ & 5.4 & 0.93 & 2380 \\
\hline & he $=0.130$ & 3.14E-08 & 5.5 & 0.96 & 2380 \\
\hline & he $=0.150$ & 3.54E-08 & 5.5 & 0.96 & 2108 \\
\hline & he $=0.170$ & $3.44 \mathrm{E}-08$ & 5.5 & 0.96 & 2170 \\
\hline
\end{tabular}

L'examen du tableau montre que les caractéristiques du milieu poreux sont peu sensibles à la hauteur d'eau $h_{e}$ du milieu externe que ce soit pour la perméabilité ou le coefficient de masse ajoutée. Plus particulièrement, la perméabilité K (et donc le coefficient de tortuosité) garde une valeur sensiblement constante, de l'ordre de $3,310^{-8} \mathrm{~m}^{2}$ quelque soit la configuration expérimentale. Cette remarque justifie a posteriori le choix fait de négliger le terme d'inertie non linéaire intervenant dans les forces résistantes de l'équation (1).

Par contre le coefficient d'instationnarité $S$ est plus sensible aux conditions expérimentales. Cette sensibilité est due au coefficient de masse ajoutée dont la valeur est de l'ordre de $0,8 \pm 25 \%$. Ce coefficient est intimement lié à la topologie de l'écoulement à l'échelle du pore et sa définition est délicate. Ce problème de dispersion du coefficient de masse ajoutée est courant en hydrodynamique lorsque l'on traite les problèmes de sillages d'obstacles soumis à un écoulement instationnaire.

\section{Champ de vitesse}

Nous avons mesuré par anémométrie laser un profil de vitesse avec une épaisseur de poreux de $50 \mathrm{~mm}$, une hauteur d'eau du milieu externe de $0,17 \mathrm{~m}$ et une période de houle de $1 \mathrm{~s}$. Pour ces mêmes valeurs nous avons déduit du modèle, les profils de vitesse théoriques.

La figure 5 présente les distributions, suivant une verticale, des composantes horizontales et verticales (valeurs maximales, minimales et moyennes sur une période) des vitesses expérimentales et théoriques.

On peut constater sur ces profils une discontinuité des vitesses horizontales accompagnée d'un saut de phase. La valeur théorique de la vitesse maximale dans le milieu poreux donne un nombre de Reynolds de l'ordre de 150.

\section{Conclusion}

Une meilleure estimation de l'écoulement dans le milieu poreux passe évidemment par une modélisation plus sophistiquée de la couche «limite» à l'interface. Dans cette couche nous ne pouvons pas ignorer la viscosité du fluide dans le milieu externe; cela rend le problème beaucoup plus complexe à résoudre théoriquement. Le modèle physique de billes sphériques n'est alors plus adapté. Le nombre de Reynolds atteint n'est pas négligeable ; même si nous pensons que le terme quadratique associé a des effets assez faibles par rapport au terme instationnaire il reste à le vérifier en essayant de le quantifier. 

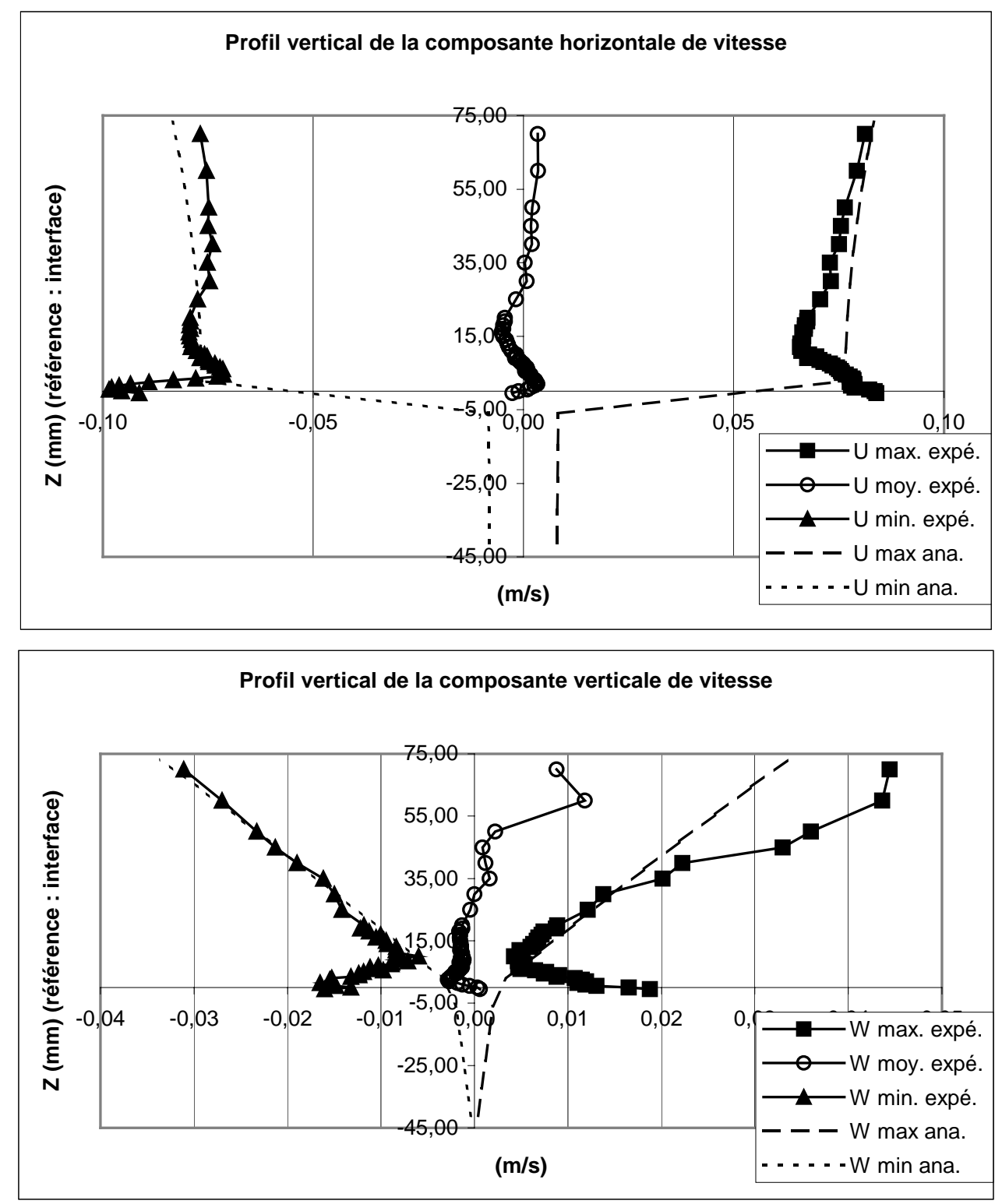

Figure 5 - Profils verticaux des composantes de vitesse

\section{Références}

1 Polubarinova-Kochina P. Ya., (1952). Theory of ground water movement (in russian), Gostekhizdat, Moscow. English transl. by R. J. M. De Wiest, (1962), Princeton University Press.

2 Brossard J., Hémon A., Rivoalen E, (2000). Improved analysis of regular gravity waves and coefficent of reflexion using one or two moving probes. Coastal Engineering 39, pp. 193212.

3 Solitt C.K., Cross R.H., (1972). Wave transmission through permeable breakwaters. $13^{\text {th }}$ Coastal Engineering Conf., vol. 3, ASCE, Vancouver, pp. 1827-1846.

4 Gu Z., Wang H., (1991). Gravity waves over porous bottoms. Coastal Engineering, 15, pp. 497-524.

5 Engelund F., (1953). On the laminar and turbulent flows of ground water through homogeneous sand. Trans. Danisch Acad. Tech. Sci., 3(4). 